\title{
A novel patient with an attenuated Costello syndrome phenotype due to an HRAS mutation affecting codon 146-Literature review and update
}

\author{
Annie Ting Gee $\stackrel{\text { Q1 }}{-}$ Chiu $^{1}$ | Gordon Ka-Chun Leung ${ }^{1}$ | Yoyo Wing-Yiu Chu ${ }^{1}$ | \\ Karen W. Gripp ${ }^{2}$ | Brian Hon- $\forall$ in Chung ${ }^{1}$
}

1 ŁKS Faculty of Medicine, Department of Paediatrics and Adoleseent Medicine, The University of Hong Kong, Hong Kong, China,

2 Division of Medical ${ }^{\mathrm{Q} 2}$ Genetics, A.I. duPont Hospital for Children, Wilmington, Delaware

Correspondence

Dr. Brian Hon-Yin Chung, tKS Faculty of Medicine, Department of Paediatries and Adoleseent Medicine, The University of Hong Kong, HKSAR. Rm 103, 1/F, New Clinical Building, Queen Mary Hospital, Hong Kong, China.

Email: bhychung@hku.hk
De novo germline mutations in HRAS cause Costello syndrome, with $>95 \%$ of the mutations causing Costello syndrome affecting amino acid position 12 (p.Gly12) or 13 (p.Gly13). We report on a patient with de novo missense mutation causing an amino acid change at codon 146 of HRAS, c.436G > C:p.Ala146Pro, who presented with subtle dysmorphic features, failure to thrive, global developmental delay, and hypertrophic obstructive cardiomyopathy. Mutations affecting codon 146 are observed in $<1 \%$ of patients with Costello syndrome. From literature search, there were only two other patients reported with mutations involving the same location. We summarized and updated their findings, and discussed evidence to show that these patients with less obvious signs of Costello syndrome may not necessarily run a more benign clinical course.

KEYWORDS

Costello syndrome, HRAS mutation, RASopathy

\section{1 | INTRODUCTION}

Costello syndrome is a distinctive rare multisystem disorder comprising of characteristic facial appearance (thin hair, coarse facies, thick eyebrow, downslanted palpebral fissures, depressed nasal bridge, low set ears, thick lips, and full cheeks), intellectual disability, growth disturbance (biphasic pattern with intrauterine overgrowth and postnatal failure to thrive that improves later in life), and tumor predisposition (Hennekam, 2003; Johnson et al., 1998; Sammon et al., 2012).

It is caused by HRAS mutations exclusively (Aoki et al., 2005). The disease-causing mutations result in amino acid substitutions that allow constitutive or prolonged HRAS activation and subsequently dysregulation of the Ras/mitogen activated protein kinase pathway (Gripp \& Lin, 2012). Over $90 \%$ of the mutations causing Costello syndrome affect amino acid position 12 (p.Gly12) or 13 (p.Gly13) (Gripp \& Lin, 1993). Among them, the three commonest mutations are p.Gly12Ser (81.3\% of total), p.Gly12Ala (7.2\%), and p.Gly12Cys (2\%). Mutations affecting position 146 are rare and estimated to be seen in less than $1 \%$ of patients (Sol-Church et al., 2009). Here, we report on a patient who presented with failure to thrive and poor feeding, and was found to have a novel p.Ala146Pro HRAS mutation by exome sequencing. We reviewed the literature on patients with mutation involving this amino acid position and obtained updated information on these patients.

\section{2 | CLINICAL REPORT}

Our patient was born full term to a non-consanguineous Chinese couple with a birth weight of $3.1 \mathrm{~kg}$ ( 25 th centile). The prenatal course was uneventful. He had poor feeding and failure to thrive, which did not improve with dietetic intervention. At 6 months of age, he weight was $4.96 \mathrm{~kg}$ (<3rd centile, 50th centile for 1.5 months), with a height of $59 \mathrm{~cm}$ ( $<3 \mathrm{rd}$ centile, 50th centile for 2.5 months), and OFC of $43 \mathrm{~cm}$ (25th centile). Initial physical examination by his pediatrician did not reveal dysmorphic features.

Laboratory investigations showed metabolic acidosis with elevated fasting lactate of $6.7 \mathrm{mmol} / \mathrm{L}$. Common mitochondrial hotspot mutation screening was negative. His initial thyroid function test was suspicious of secondary hypothyroidism with thyroid stimulating hormone of $0.35 \mathrm{mIU} / \mathrm{L}$ and free thyroxine of $9.5 \mathrm{pmol} / \mathrm{L}$. However, it subsequently normalized and the thyroid-releasing-hormone 

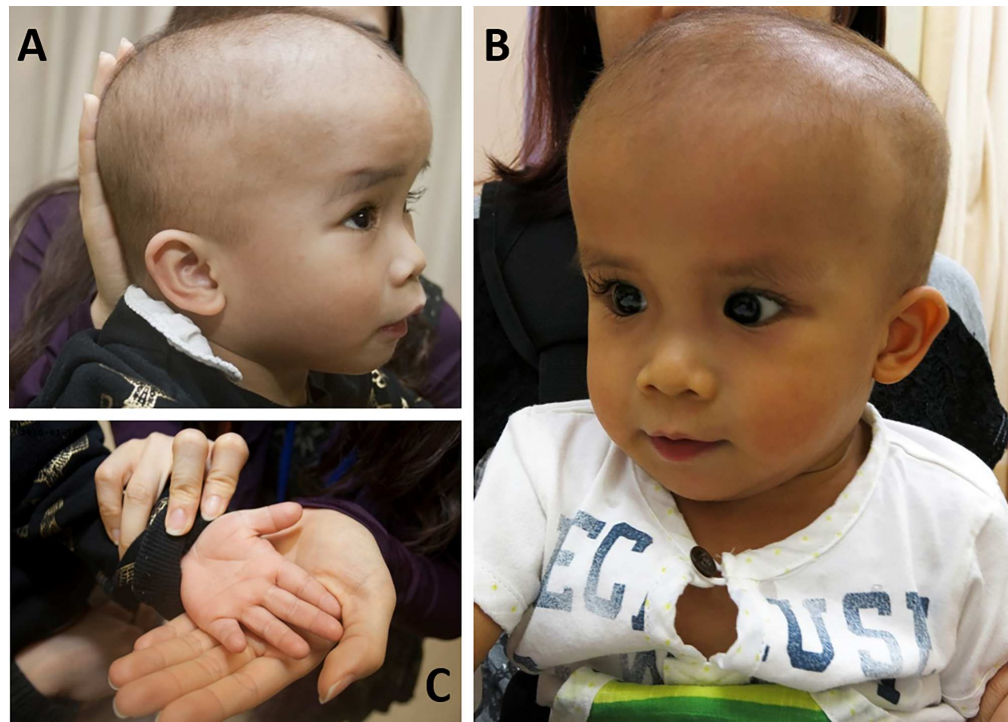

FIGURE 1 Q3 Clinical photographs of the index patient with p.Ala146Pro mutation of HRAS. A) Profile view of his face (taken at 36 months) and B) Frontal view of his face (taken at 33 months). Note the fontal bossing, sparse and short hair, long eyelashes, deep set eyes, low set ears, short nasal tip, long philtrum and the absence of full cheeks and full lips. C) Clinical photographs of his hand with absence of deep palmer crease (taken at 36 months)

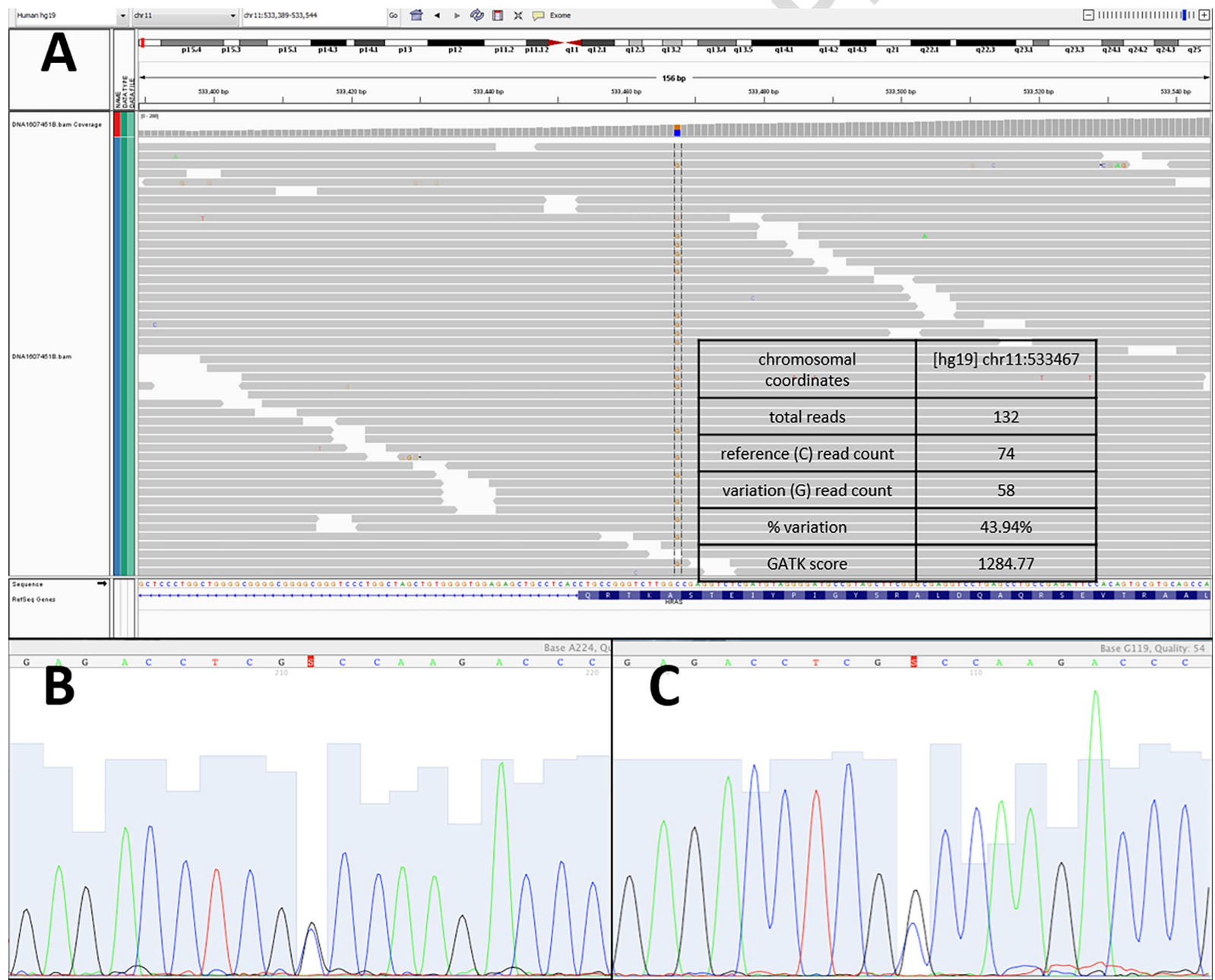

FIGURE 2 Screenshot of exome sequencing data of the index patient, showing a mutation in HRAS: c.436G>C:p,Ala146Pro (Figure 2A); subsequent Sanger validation confirmed the findings in the index patient's peripheral blood sample (Figure 2B) and buccal swab (Figure 2C), showing no evidence of mosaicism 


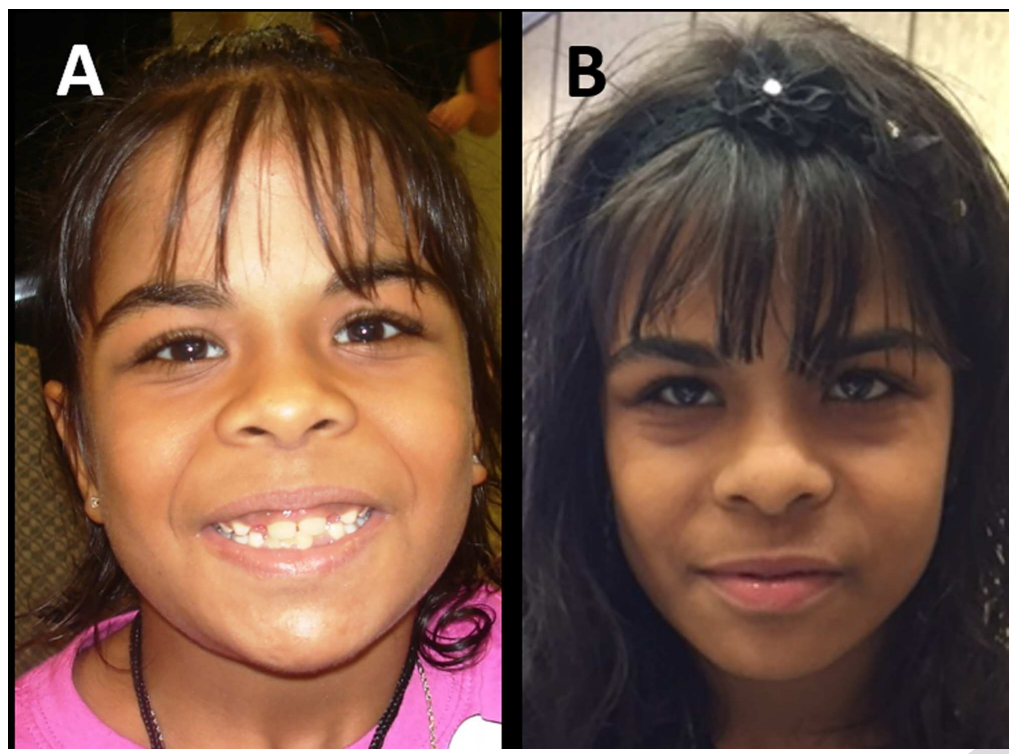

FIGURE 3 Clinical photographs of the patient with p.Ala146Val mutation of HRAS as reported[Gripp et al., 2008]. A) Frontal view at age 9 years. B) Frontal view at 13 years

stimulation test was normal. There was transient neutropenia with absolute neutrophil count of $0.25-0.5 \times 10^{\wedge} 9 / \mathrm{L}$ which resolved after an episode of intercurrent illness. Other evaluations were essentially unremarkable with normal biotinidase level, carnitine, and acylcarnitine profile, dried blood spot test for amino acid and urine for organic acid. Brain MRI at 9 months showed an arachnoid cyst at the left posterior cranial fossa measuring $1.2 \mathrm{~cm}$. It was repeated at 2.5 years showing similar findings.

He had moderate global developmental delay at 2.5 years and was referred for genetic evaluation. Physical assessment showed frontal bossing with sparse and short hair, long eyelashes, deep set eyes, low set ears, short nasal tip, long philtrum, dry skin and a left sided inguinal hernia. His cheeks and lips were not full, there was no deep palmer crease, no evidence of pectus deformity and no kyphoscoliosis (Figure 1).

Initial genetic evaluation including karyotype, chromosomal microarray, and methylation-specific multiplex ligation-dependent probe amplification of chromosome 11, were normal. Singleton exome sequencing (Mendelian inherited disorder panel, performed by Genome Diagnostics Nijmegen) identified a mutation in HRAS: c.436G>C:p.Ala146Pro, and subsequent parental testing confirmed its de novo origin. A screenshot of the exome sequencing data and the chromatogram of the subsequent Sanger validation showing the mutation is shown in Figure 2A and B. Sanger confirmation using DNA from the patient's buccal mucosa was also performed (Figure $2 \mathrm{C}$ ), showing similar findings as the peripheral blood sample and was not suggestive of mosaicism.

He was found to have a cardiac murmur at 3 years. Echocardiogram showed asymmetrical left ventricular hypertrophy which was more severe over the interventricular septum, systolic anterior motion of the mitral valve as well as left ventricular outflow tract obstruction with subvalvular aortic stenosis of $25-50 \mathrm{mmHg}$, suggestive of hypertrophic obstructive cardiomyopathy (HCM). He was monitored conservatively with serial echocardiogram.
His global developmental delay persisted despite intensive multidisciplinary training. At 4 years, he was walking fast, could scribble, and build a tower of two bricks. He was able to identify five body parts and had multiple single words. In terms of self-care skills, he could feed himself with a spoon and indicate toilet needs. Overall, his developmental profile was evenly distributed with a developmental quotient below 50 .

\section{1 | Literature review and updated information on previously reported patients}

Only two other patients have been reported with HRAS mutations involving codon 146 (Gripp et al., 2008; Zampino et al., 2007). Gripp et al. (2008) reported a 5.5 years old girl with a p.Ala146Val mutation, with mildly coarse facial features, short nasal tip, long philtrum, and mildly prominent ears without strikingly deep palmer creases. She also had global developmental delay, feeding difficulties requiring gastrostomy placement, history of treatment resistant tachycardia, growth hormone deficiency, and HCM requiring myomectomy (Gripp et al., 2008). Update from her primary care team revealed that she has not developed further cardiac hypertrophy while on metoprolol, but subsequent echocardiography noted mitral regurgitation at 8 years and ventricular septal defect with mild aortic regurgitation at 10 years. Brain imaging showed mild cerebellar tonsillar herniation (Gripp, Hopkins, Doyle, \& Dobyns, 2010). Osteoporosis was treated with calcium and vitamin D supplements. Coxa valga did not require medical intervention (Detweiler, Thacker, Hopkins, Conway, \& Gripp, 2013). She was in puberty had no menarche by age 14.5 years. Neuropsychological evaluation at 9 years in her native language showed a full scale IQ of 50 (verbal 53, non-verbal 51). She was originally studying in a mainstream school at grade level. However, after a move and change in the primary language spoken in school, learning difficulties resulted in placement in a school for individuals with moderate intellectual 
TABLE 1 Comparing the Costello Syndrome Severity Scoring System (CSSSS) Score [McCormick et al., 2013] in the 2 reported patients with HRAS p.Ala146 mutation and the index patient

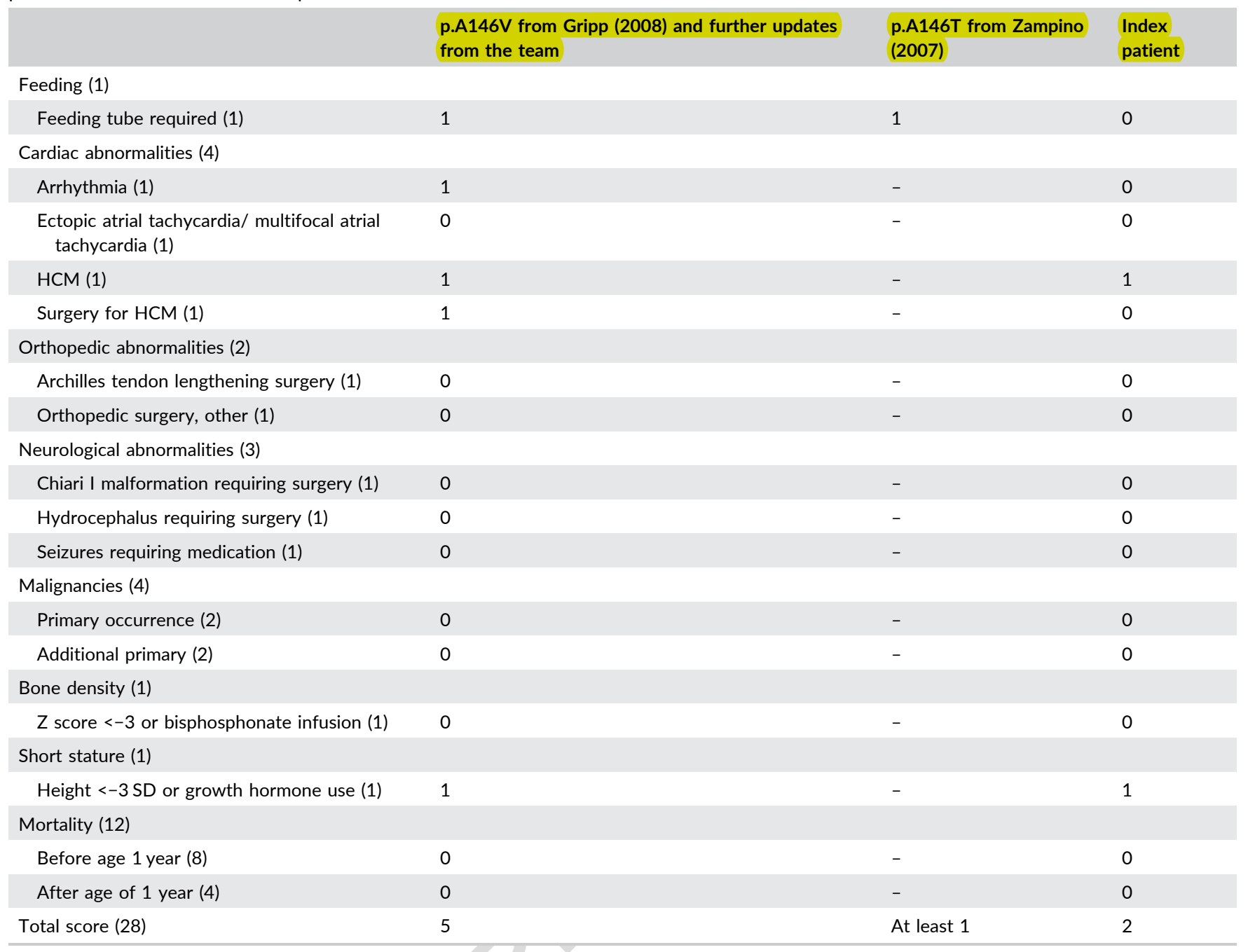

"_" indicates information not available.

disability, where she received an individualized education plan and had a behavior specialist for a diagnosis of anxiety. At 14 years, she attended a vocational school program and hoped to become an early childhood teacher's assistant. Her dysmorphic features has remained subtle (Figure $3 \mathrm{~A}$ and $\mathrm{B}$ ).

Zampino et al. (2007) reported another patient with p.Ala146Thr mutation, with normal birth parameters, microcephaly, sparse thin but not curly hair, normally located ears, less compromised growth, and minor skin and joint involvement. Information regarding her systemic involvement was lacking. Unfortunately, we were not able to obtain further updates about the patient's current condition.

\section{3 | DISCUSSION}

Costello syndrome is a RASopathy with multisystemic involvement including cardiac, orthopedic, neurological, growth, and musculoskeletal abnormalities as well as a predisposition for malignancies (Gripp \& Lin, 2012). Most patients with Costello syndrome harbor a mutation at codon 12 of the HRAS, the most common one being p.Gly12Ser, accounting for around 80\% (Kerr et al., 2006; McCormick et al., 2013). Alteration of glycine at position 12 or 13 of HRAS accounts for more than $90 \%$ cases of Costello syndrome. The mutated protein becomes bound to GTP in a constitutive or prolonged manner and activates multiple downstream pathway including MAPK, PI-3 kinase, and RalGDS (Aoki et al., 2005). This leads to altered cell growth, proliferation, and cell death, which is consistent with many of the clinical features seen in Costello syndrome.

Despite the overwhelming predominance of codon 12 or 13 related HRAS mutation, other mutations have been reported, as in the patient with substitution of alanine at position 146 by proline. To our knowledge, this is the third reported patient with Costello syndrome involving position 146 of HRAS. This residue is located within the G3 motif and is totally conserved in the RAS superfamily (Mitin, Rossman, \& Der, 2005). It is involved in the binding to the purine ring of GTP/ GDP and interacts directly with the guanine base (Wittinghofer \& Waldmann, 2000). Substitutions are predicted to destabilize HRAS binding to either GTP or GDP, but since GTP has a higher 
concentration in the cytoplasm it will shift the equilibrium toward the GTP bound active form (Zampino et al., 2007), acting in much the same way as mutations in the position 12/13 hotspot.

Individuals with various mutations have an increased predisposition to malignancy, severe HCM, neonatal mortality, neurological abnormality, and other complications (Kerr et al., 2006; McCormick et al., 2013). Patients with the p.Gly12Cys mutation have less coarse facial features subjectively and loose anagen hair with a matted appearance. They also have significantly less multifocal atrial tachycardia, ulnar wrist deviation, papilloma, and short stature (Gripp et al., 2011). The expression of various downstream pathways, however, were not shown to be different between genotypes in functional studies (Gremer et al., 2010), and it is postulated that the observed genotype-phenotype correlation may be secondary to cell type specific effects (Gripp et al., 2011). However, genotypephenotype correlation of rare mutation like those involving codon 146 is given less attention simply because they are difficult to study due to the rarity.

Comparing the three patients with HRAS mutations affecting codon Ala 146, consistently the facial gestalt and physical findings seems to be milder than patients with mutation at codon $12 / 13$ hotspot and this includes less coarse facies, absence of thick lip, and deep-palmer crease. Table 1 compared the clinical severity of the three patients with codon 146 mutation, using the Costello Syndrome Severity Scoring System (CSSSS) (McCormick et al., 2013). As stated above, updated clinical information of the patient with p.Ala146Thr is lacking. However, our patient with $\mathrm{p}$. Ala146Thr scored 2, which is equivalent to the mean CSSSS score in patients with p.Gly12Ser mutation in their early childhood, while the patient with p.Ala146Val already had a score of 5 at age 5 years, which is above the mean score of 3.743 in children with $p$. Gly12Ser mutation at 10 years old (McCormick et al., 2013). Therefore it is important to recognize that these patients with less obvious signs of Costello syndrome may not necessarily run a more benign clinical course.

Further management of this child would involve continual medical surveillance for development and growth, malignancy, neurological, and orthopedic complications (Gripp et al., 2010). Patients with Costello syndrome are prone to development of malignancy, especially rhabdomyosarcoma, neuroblastoma, bladder cell carcinoma, and pituitary adenoma (Lin et al., 2011). In order to determine the malignancy potential of the HRAS p.Ala146 mutation, we searched the COSMIC database (Bamford et al., 2004) in a similar way as Gripp et al. (2008) for somatic mutations involving the HRAS as well as the highly homologous KRAS in human cancer tissues. In a total of 25,736 and 26,421 samples analyzed for HRAS and KRAS principal transcript, 196 and 2,816 mutations were identified for HRAS and KRAS, respectively. Whereas no mutation was identified in position 146 of HRAS, 68 mutations were found involving position 146 of KRAS, with 52 cases of p.Ala146Thr, 13 cases of p.Ala146Val, and three cases of p.Ala146Pro. Such results support the need for vigilance regarding tumor surveillance. It should be noted, however, that cancer surveillance in Costello syndrome has been proven to be challenging, for example, routine screening for neuroblastoma with urine for catecholamine is not effective, as children with Costello syndrome are known to have higher urinary excretion of catecholamines compared to the normal population (Gripp, Kawame, Viskochil, \& Nicholson, 2004).

Another important field of surveillance important is brain imaging. Brain malformation is highly prevalent in Costello syndrome, and most of it pertains to postnatal onset posterior fossa crowding with cerebellar tonsillar herniation, which may progress with time and lead to hydrocephalus, Chiari malformation, and syrinx formation (Gripp et al., 2010).

Yet another field of surveillance is growth. While Costello syndrome is associated with failure to thrive per se, nearly half of the patients also have concomitant partial growth hormone deficiency (Rauen et al., 2008) and there have been multiple case reports on the successful use with growth hormone (Blachowska et al., 2016; Stein, Legault, Daneman, Weksberg, \& Hamilton, 2004). However, growth hormone replacement need be considered with caution in the setting of HCM. Even though no significant difference in progression of HCM was noted in children receiving or not receiving growth hormone replacement (Rauen et al., 2008), re-emergence of previously resolved HCM during growth hormone replacement has been reported (Kobayashi, Cook, \& Williams, 2010). Rapid progression of embryonic rhabdomyosarcoma has also been reported while a patient with Costello syndrome was put on growth hormone replacement (Kerr et al., 2003). Thus a thorough evaluation should be performed before commencement of recombinant growth hormone therapy.

In summary, we report on a patient with Costello syndrome with the novel position 146 mutation, p.Ala146Pro, in HRAS. The physical features suggestive of Costello syndrome were noted to be less classical. Detailed genotype-phenotype analysis is not possible due to the rarity of position 146 mutation, but current evidence suggests the same health surveillance as in other patients with Costello syndrome should be offered. Long-term follow up of these patients will be necessary for more tailor-made recommendations.

\section{ACKNOWLEDGMENT}

We would like to thank the Hong Kong Society for the Relief of Disabled Children for financing the genetic testing of the index patient.

Disclosures: We, the author and co-authors of the case report, have no actual or potential conflicts of interest, including any financial, personal, or other relationships with people or organizations regarding the work.

\section{REFERENCES}

Aoki, Y., Niihori, T., Kawame, H., Kurosawa, K., Ohashi, H., Tanaka, Y., ... Matsubara, Y. (2005). Germline mutations in HRAS proto-oncogene cause Costello syndrome. Nature Genetics, 37, 1038-1040.

Bamford, S., Dawson, E., Forbes, S., Clements, J., Pettett, R., Dogan, A., .. . Stratton, M. (2004). The COSMIC (Catalogue of Somatic Mutations in Cancer) database and website. British journal of cancer, 91, 355-358.

Blachowska, E., Petriczko, E., Horodnicka-Jozwa, A., Skorka, A., Pelc, M., Krajewska-Walasek, M., \& Walczak, M. (2016). Recombinant growth hormone therapy in a girl with Costello syndrome: A 4-year observation. Italian Journal of Pediatrics, 42, 10.

Detweiler, S., Thacker, M.M., Hopkins, E., Conway, L., \& Gripp, KW. (2013). Orthopedic manifestations and implications for individuals with Costello syndrome. American Journal of Medical Genetics Part A, 161A, 1940-1949. 
children with Noonan, Costello, and Eardio facio-cutaneous syndromes. British Journal of Cancer, 112, 1392-1397. S., Tartaglia, M., ... Rosenberger, G. (2010). Duplication of Glu37 in the switch I region of HRAS impairs effector/GAP binding and underlies Costello syndrome by promoting enhanced growth factor-dependent MAPK and AKT activation. Human Molecular Genetics, 19, 790-802.

Gripp, K.W., Hopkins, E., Doyle, D., \& Dobyns, W.B. (2010). High incidence of progressive postnatal cerebellar enlargement in Costello syndrome: Brain overgrowth associated with HRAS mutations as the likely cause of structural brain and spinal cord abnormalities. American Journal of Medical Genetics Part A, 152A, 1161-1168.

Gripp, K.W., Hopkins, E., Sol-Church, K., Stabley, D.L., Axelrad, M.E., Doyle, D., ... Lin, A.E. (2011). Phenotypic analysis of individuals with Costello syndrome due to HRAS p.G13C. American Journal of Medical Genetics Part A, 155A, 706-716.

Gripp, K.W., Innes, A.M., Axelrad, M.E, Gillan, T.L., Parboosingh, J.S., Davies, C, ... Sol-Church, K. (2008). Costello syndrome associated with novel germline HRAS mutations: An attenuated phenotype? American Journal of Medical Genetics Part A, 146A, 683-690.

Gripp, K.W., Kawame, H., Viskochil, D.H., \& Nicholson, L. (2004). Elevated catecholamine metabolites in patients with Costello syndrome. American Journal of Medical Genetics Part A, 128A, 48-51.

Gripp, K.W., \& Lin, A.E. (1993), Costello Q4 syndrome. In: R.A. Pagon, M.P. Adam, H.H. Ardinger, S.E. Wallace, A. Amemiya, L.J.H. Bean, T.D. Bird, C.T. Fong, H.C. Mefford, R.J.H. Smith, K. Stephens, (Eds). GeneReviews(R). Seattle $(W A)_{2}$

Gripp, K.W., \& Lin, A.E. (2012). Costello syndrome: A Ras/mitogen activated protein kinase pathway syndrome (rasopathy) resulting from HRAS germline mutations. Genetics in Medicine, 14, 285-292.

Hennekam, R.C. (2003). Costello syndrome: An overview. American Journal of Medical Genetics Part C Seminars in Medical Genetics, 117C, 42-48.

Johnson, J.P., Golabi, M., Norton, M.E., Rosenblatt, R.M., Feldman, G.M., Yang, S.P., ... Carey, J.C. (1998). Costello syndrome: Phenotype, natural history, differential diagnosis, and possible cause. Journal of Pediatrics, 133, 441-448.

Kerr, B., Delrue, M.A., Sigaudy, S., Perveen, R., Marche, M., Burgelin, I., .. . Black, G. (2006). Genotype-phenotype correlation in Costello syndrome: HRAS mutation analysis in 43 cases. Journal of Medical Genetics, 43, 401-405.

Kerr, B., Einaudi, M.A., Clayton, P., Gladman, G., Eden, T., Saunier, P., ... Philip, N. (2003). Is growth hormone treatment beneficial or harmful in Costello syndrome? Journal of Medical Genetics, 40, 74.

Kobayashi, D., Cook, A.L., \& Williams, D.A. (2010). Progressively worsening hypertrophic cardiomyopathy in a child with newly diagnosed Costello syndrome while receiving growth hormone therapy. Cardiology Young, 20, 459-461.

Kratz, C.P., Franke, L., Peters, H., Kohtschmidt, N., Kazmierczak, B., Finckh, U., ... Zenker, M. (2015). Cancer $05_{\text {spectrum and frequency ameng }}$
Lin, A.E., Alexander, M.E., Colan, S.D., Kerr, B., Rauen, K.A., Noonan, J., .. . Gripp, K.W. (2011). Clinical, pathological, and molecular analyses of cardiovascular abnormalities in Costello syndrome: A Ras/MAPK pathway syndrome. American Journal of Medical Genetics Part A, 155A, 486-507.

McCormick, E.M., Hopkins, E., Conway, L., Catalano, S., Hossain, J., SolChurch, K., ... Gripp, K.W. (2013). Assessing genotype-phenotype correlation in Costello syndrome using a severity score. Genetics in Medicine, 15, 554-557.

Mitin, N., Rossman, K.L., \& Der, C.J. (2005). Signaling interplay in Ras superfamily function. Current Biology, 15, R563-R574.

Rauen, K.A., Hefner, E., Carrillo, K., Taylor, J., Messier, L., Aoki, Y., ... Schoyer, L. (2008). Molecular aspects, clinical aspects and possible treatment modalities for Costello syndrome: Proceedings from the 1st International Costello Syndrome Research Symposium 2007. American Journal of Medical Genetics Part A, 146A, 1205-1217.

Sammon, M.R., Doyle, D., Hopkins, E., Sol-Church, K., Stabley, D.L., McGready, J., ... Gripp, K.W. (2012). Normative growth charts for individuals with Costello syndrome. Am J Med Genet Part A, 158A, 2692-2699.

Sol-Church, K., Stabley, D.L., Demmer, L.A., Agbulos, A., Lin, A.E., Smoot, L., ... Gripp, K.W. (2009). Male-to-male transmission of Costello syndrome: G12S HRAS germline mutation inherited from a father with somatic mosaicism. American Journal of Medical Genetics Part A, 149A, 315-321.

Stein, R.I., Legault, L., Daneman, D., Weksberg, R., \& Hamilton, J. (2004). Growth hormone deficiency in Costello syndrome. American Journal of Medical Genetics Part A, 129A, 166-170.

Wittinghofer, A., \& Waldmann, H. (2000). Ras-a molecular switch involved in tumor formation. Angewandte Chemie International Edition, 39, 4192-4214.

Zampino, G., Pantaleoni, F., Carta, C., Cobellis, G., Vasta, I., Neri, C., ... Tartaglia, M. (2007). Diversity, parental germline origin, and phenotypic spectrum of de novo HRAS missense changes in Costello syndrome. Human Mutatation, 28, 265-272.

How to cite this article: Chiu ATG, Leung GK, Wing Yiu Chu $Y_{2}$ Gripp KW, and Chung BH. A novel patient with an attenuated Costello syndrome phenotype due to an HRAS mutation affecting codon 146-Literature review and update. Am J Med Genet Part A. 2016;9999:1-6. doi:10.1002/ajmg.a.38118. 